\title{
Importance of Handgrip Strength as a Health Indicator in the Elderly
}

\author{
Soo Young Kim* \\ Department of Family Medicine, Kangdong Sacred Heart Hospital, College of Medicine, Hallym University, Seoul, Korea
}

\section{See original paper on $\mathbf{3 8}$}

A decline in physical function is a natural phenomenon associated with aging. However, as it is also associated with an increased risk of falls, health care use, levels of dependence, and premature death, ${ }^{1)}$ it is a major public health issue. Handgrip strength (HGS) is a commonly used measure of physical function. In recent years, there has been an increase in literature investigating the association between HGS and health outcomes. There are reports of a correlation between HGS and dyslipidemia ${ }^{2)}$ cognitive function, ${ }^{3)}$ and diabetes. Most of the studies to date have addressed a single disease endpoint; some studies report an association between baseline HGS and mortality risk. However, there are few studies on the relationship between the degree of change in HGS and the risk of mortality. Additionally, there are no studies conducted on a Korean population.

In the present issue, Bae et al. ${ }^{3)}$ investigated the association between HGS and mortality among older adults in Korea. In particular, this study investigated the trajectories of HGS using the 2006-2016 Korean Longitudinal Study of Aging. The results of the study revealed that, compared with the highest HGS group, the adjusted hazard ratio of all-cause-mortality in the group with the lowest HGS was 3.46 (95\% confidence interval [CI], 2.17-6.69) in men, and 2.69 (95\% CI, 1.39-5.21) in women. In addition, the authors identified four distinct trajectory groups of HGS among men, and three among women. In both men and women, the risk of mortality was inversely proportional to the HGS.

As the risk of all-cause mortality in both men and women, increased with rapid decline in hand grip strength, it may be inferred that measuring HSG in the elderly is helpful in measuring health status.
A sedentary life style has been suggested as one of the factors related to a decline in HGS. ${ }^{4)}$ However, a clear mechanism to explain the relevance of HGS scores to these health outcomes has not been elucidated. In order to implement intervention programs, further research may be required.

\section{CONFLICT OF INTEREST}

No potential conflict of interest relevant to this article was reported.

\section{ORCID}

Soo Young Kim: https://orcid.org/0000-0002-3205-9408

\section{REFERENCES}

1. Soysal P, Hurst C, Demurtas J, Firth J, Howden R, Yang L, et al. Handgrip strength and health outcomes: Umbrella review of systematic reviews with meta-analyses of observational studies. J Sport Health Sci 2020 Jun 19 [Epub]. https://doi.org/ 10.1016/j.jshs.2020.06.009.

2. Kim BM, Yi YH, Kim YJ, Lee SY, Lee JG, Cho YH, et al. Association between relative handgrip strength and dyslipidemia in Korean adults: findings of the 2014-2015 Korea National Health and Nutrition Examination Survey. Korean J Fam Med 2020;41:404-11.

3. Bae KH, Jo YH, Lee DR, Lee J. Trajectories of handgrip strength and their associations with mortality among older adults in Korea: analysis of the Korean Longitudinal Study of Aging. Korean J Fam Med 2021;42:38-46.

4. Lee SY, Son DH, Lee YJ. Relationship between sedentary time and handgrip strength in healthy Korean women: Korea National Health and Nutrition Examination Survey 2014-2016. Korean J Fam Med 2020;41:422-6.

*Corresponding Author: Soo Young Kim https://orcid.org/0000-0002-3205-9408 